\title{
Dickkopf-related protein 1 induces angiogenesis by upregulating vascular endothelial growth factor in the synovial fibroblasts of patients with temporomandibular joint disorders
}

\author{
SHENG-JUN JIANG ${ }^{1}$, WEI LI ${ }^{1}$, YING-JIE LI ${ }^{1}$, WEI FANG ${ }^{2}$ and XING LONG ${ }^{2}$ \\ ${ }^{1}$ The State Key Laboratory Breeding Base of Basic Science of Stomatology (Hubei-MOST) and \\ Key Laboratory of Oral Biomedicine Ministry of Education, School and Hospital of Stomatology; \\ ${ }^{2}$ Department of Oral and Maxillofacial Surgery, School and Hospital of Stomatology, \\ Wuhan University, Wuhan, Hubei 430079, P.R. China
}

Received September 26, 2014; Accepted June 17, 2015

DOI: $10.3892 / \mathrm{mmr} .2015 .4101$

\begin{abstract}
Angiogenesis has an important role in the progression of temporomandibular joint disorders (TMD). The aim of the present study was to explore the association between dickkopf-related protein 1 (DKK-1) and angiogenesis in TMD. The expression levels of DKK-1 and vascular endothelial growth factor (VEGF) were quantified by an ELISA assay of the synovial fluid from patients with TMD. The correlation between DKK-1 and VEGF was analyzed by Pearson correlation test. Synovial fibroblasts were isolated from patients with TMD and were subsequently treated with recombinant human DKK-1, anti-DKK-1 antibody, hypoxia inducible factor-1 $\alpha$ (HIF-1 $\alpha$ ), or small interfering RNA (siRNA). The expression levels of DKK-1, HIF- $1 \alpha$, and VEGF were subsequently quantified. The present study also investigated the effects of DKK-1 on the migration of human umbilical vein endothelial cells (HUVEC). Increased expression levels of DKK-1 were concordant with increased expression levels of VEGF in the synovial fluid from patients with TMD. In the synovial fibroblasts, DKK-1 increased the expression levels of VEGF, and promoted HIF-1 $\alpha$ nuclear localization. In addition, DKK-1 induced HUVEC migration, and HIF- $1 \alpha$ siRNA inhibited DKK-1-induced cell migration. The results of the present study indicate that DKK-1 is associated with angiogenesis in the synovial fluid of patients with TMD. Furthermore, HIF-1 $\alpha$ may be associated with DKK-1-induced HUVEC activation.
\end{abstract}

Correspondence to: Professor Xing Long, Department of Oral and Maxillofacial Surgery, School and Hospital of Stomatology, Wuhan University, 237 Luoyu Road, Wuhan, Hubei 430079, P.R. China E-mail: longxing_china@hotmail.com

Key words: dickkopf-related protein 1, temporomandibular disorders, hypoxia inducible factor-1 $\alpha$, vascular endothelial growth factor, angiogenesis

\section{Introduction}

Temporomandibular joint disorders (TMD) are one of the most prevalent types of joint disease, which cause persistent and recurrent joint pain with eventual loss of joint function (1). The methods of diagnosis and treatment of TMD have improved in the past three decades; however, the cellular and molecular mechanisms underlying the initiation and progression of TMD remain to be elucidated (2). Interleukin-1 $\beta$ (IL-1 $\beta)$ is an important cytokine that contributes to the severe inflammatory reactions observed in TMD (3). Histopathological studies have demonstrated that the synovial membranes of patients with TMD exhibit inflammatory cell infiltration, hyperplasia, and high-density vascularization $(4,5)$. In addition, it has been suggested that inflammation-induced angiogenesis in the synovial membranes has an important role in the progression of TMD (6).

Angiogenesis is a common process associated with the induction of cancer and other diseases (7). Numerous molecules are activated during the complex process of angiogenesis in the synovial membrane $(7,8)$. Our previous studies demonstrated that vascular endothelial growth factor (VEGF), a vascular permeability factor and selective endothelial mitogen, accumulates in the synovial membrane and fluid during the inflammatory response of TMD $(9,10)$. Previous in vivo and in vitro studies have reported that hypoxia inducible factor- $1 \alpha$ (HIF-1 $\alpha$ ) is activated in the synovial membrane of patients with TMD $(9,11)$, and implicated in the regulation of important aspects of angiogenesis $(12,13)$. Although the expression levels of angiogenic factors such as HIF-1 $\alpha$ and VEGF have been shown to be increased in the synovium of joint diseases, the mechanisms that underlie increased angiogenesis, specifically in the case of TMD, have yet to be elucidated.

Dickkopf-related protein 1 (DKK-1) is a secreted protein, which inhibits the $\mathrm{Wnt} / \beta$-catenin signaling pathway, and is implicated in various developmental and physiological processes $(14,15)$. A previous study demonstrated that DKK-1 promotes angiogenesis during development, tumorigenesis, and inflammation (16). In addition, DKK-1 has been shown 
to enhance the angiogenic properties of human endothelial colony-forming cells, and increase tumoral angiogenesis in breast cancer (17). In osteoarthritic knee joints, DKK-1 was associated with angiogenesis and cartilage matrix proteinase secretion (15). However, DKK-1 expression and the biological role of DKK-1 in the synovium of TMD are, to the best of our knowledge, rarely studied.

In the present study, the expression of DKK-1 was determined in synovial tissues from a cohort of patients with TMD and from healthy patients. The association between DKK-1 and VEGF was also examined. In addition, the effects of DKK-1 on the angiogenesis in the was investigated in vitro.

\section{Materials and methods}

Patients. Synovial tissue samples were obtained from the Department of Oral and Maxillofacial Surgery, School and Hospital of Stomatology, Wuhan University (Wuhan, China). All experiments and synovial tissue sample collections were performed in accordance with the Helsinki Declaration of 1975, and approved by the institute review board of the Ethics Committee of the Hospital of Stomatology, Wuhan University. Written informed consent was obtained from all patients. The patients with TMD were diagnosed and classified according to the Research Diagnostic Criteria for TMD $(18,19)$. Patients who received any oral medication within the previous 6 months, or any other intra-articular treatment, were excluded from the present study. The clinical data and descriptive characteristics of the patients are shown in Table I. Synovial tissue samples were collected bilaterally from six patients, and unilaterally from 44 patients. All of the patients were sorted into anterior disc displacement with reduction (ADDwR), anterior disc displacement without reduction (ADDw/oR), and osteoarthritis (OA) TMD groups, according to their clinical characteristics, radiographic examination and arthrography. The ADDwR and ADDw/oR subgroups formed the anterior disc displacement (ADD) group. A total of seven samples from healthy volunteers were included as the control group. The synovial fluid harvesting method was carried out as previously described (20). Briefly, the patients received 2\% lidocaine (Chengdu No.1 Pharmaceutical Group Co. Ltd., Chengdu, China) in the preauricular region by subcutaneous infiltration, then $2 \mathrm{ml}$ saline solution was injected into the superior joint space. The synovial fluid was then mixed once the patient opened and closed his or her mouth. The saline solution was subsequently aspirated and collected, and an arthrography examination was carried out at the same time. The synovial tissue samples from the control subjects were collected in a similar manner, except that they did not receive temporomandibular joint (TMJ) arthrography. The synovial tissue samples were centrifuged at $4^{\circ} \mathrm{C}(1,000 \mathrm{x} \mathrm{g}, 5 \mathrm{~min})$ to remove the cell and tissue debris, prior to being stored at $-70^{\circ} \mathrm{C}$.

ELISA. The concentration levels of DKK-1 and VEGF in the synovial fluid from the patients with TMD were measured using a Human DKK-1 ELISA kit (Wuhan ColorfulGene Biological Technology Co., Ltd, Wuhan, China) and a Human VEGF ELISA kit (Pierce Biotechnology, Inc., Rockford, IL, USA) according to the manufacturer's instructions. The detailed methods have previously been described (20).
Cell culture and reagents. Synovial fibroblasts were cultured from the synovial tissue samples of four patients $(24,24$, 28 and 30 years-old) from the OA groups, as previously described (21). The cells were maintained in Dulbecco's modified Eagle's medium (DMEM) supplemented with $20 \%$ fetal bovine serum (FBS) (both from Thermo Fisher Scientific Inc., Beijing, China), in a humidified atmosphere containing $95 \%$ air and $5 \% \mathrm{CO}_{2}$ at $37^{\circ} \mathrm{C}$. Cell passages $3-8$ were used in the present study. Pooled HUVECs were purchased from Lonza (Walkersville, MD, USA), and grown in endothelial growth medium 2 (Lonza), with passages 3-8 being used in the present study. IL-1 $\beta$, DKK-1, human DKK-1 affinity purified rabbit polyclonal antibody (DKK-1 inhibitor), and recombinant human DKK-1 (rhDKK-1) were purchased from R\&D systems, Inc. (Minneapolis, MN, USA). The rabbit monoclonal anti-HIF-1 $\alpha$ (Epitomics Inc., Burlingame, CA, USA; ab190197; 1:1,000) and rabbit polyclonal anti-VEGF antibodies (GeneTex Inc., Irvine, CA, USA; GTX102643; 1:1,000) were obtained from Cell Signaling Technologies, Inc. (Danvers, MA, USA). Rabbit monoclonal DKK-1 was purchased from Abcam (Abcam, Cambridge, CA, USA; ab109416; 1:1000). Anti-GAPDH was purchased from Santa Cruz Biotechnology Biotechnology, Inc. (Dallas, TX, USA).

Transient transfection with small interfering RNA (siRNA). siRNA specific for human HIF-1 $\alpha$ and a non-specific control siRNA (scramble) were purchased from Shanghai GenePharma Co., Ltd. (Shanghai, China). HIF-1 $\alpha$ siRNA scramble siRNA: 5'-UAGCGACUAAACACAUCAA-3'; HIF-1a si1: 5'-GCCGCUCAAUUUAUGAAUATT-3'; and HIF-1a si2: 5'-CCAGUUAUGAUUGUGAAGUUA-3'. The cells were grown to $60-80 \%$ confluence in 12 -well plates and were transfected using Lipofectamine ${ }^{\circledR} 2000$ (Invitrogen Life Technologies, Carlsbad, CA, USA). The HIF-1 $\alpha$ and scramble siRNA $(60 \mu \mathrm{mol})$ were transfected into the cells with Lipofectamine ${ }^{\circledR} 2000$, according to the manufacturer's instructions. All experiments were performed $24 \mathrm{~h}$ post-transfection. Total RNA was extracted $48 \mathrm{~h}$ post-transfection, and protein was extracted $72 \mathrm{~h}$ post-transfection.

Western blotting. Western blot analysis was performed as described previously (9). Briefly, the synovial fibroblasts were treated with IL-1 $\beta$ or rhDKK-1 in DMEM supplemented with $2 \%$ FBS for $24 \mathrm{~h}$. The cells were then lysed using M-PER Mammalian Protein Extraction buffer (Pierce Biotechnology, Inc., Rockford, IL, USA) containing protease inhibitor tablets (complete, EDTA-free; Roche Applied Science, Shanghai, China), and the cell extracts were separated by 10 or $12 \%$ SDS-PAGE, prior to being transferred onto polyvinylidene fluoride membranes (EMD Millipore, Billerica, MA, USA). The membranes were blocked with $5 \%$ skimmed milk for $1 \mathrm{~h}$, and subsequently incubated overnight at $4^{\circ} \mathrm{C}$ with the corresponding primary antibodies, in $5 \% \mathrm{w} / \mathrm{v}$ bovine serum albumin (Beyotime Institute of Biotechnology, Nantong, China), followed by incubation with a horseradish peroxidase-conjugated secondary antibody (Pierce Biotechnology, Inc.). The blots were then developed using the West Pico Super Enhanced Chemiluminescence Detection kit (Thermo Fisher Scientific Inc., Waltham, MA, USA). GAPDH was used as a loading control and was detected on the same membrane. 
Table I. Patients and TMD characteristics.

\begin{tabular}{lcccccc}
\hline Group & No. of patients & Female & Male & Age (years) & Mean age (years) & No. of TMJs \\
\hline Control & 7 & 4 & 3 & $25-34$ & 27.3 & 7 \\
ADDwR & 18 & 10 & 8 & $17-42$ & 29.5 & 22 \\
ADDw/oR & 20 & 11 & 9 & $18-53$ & 31.7 & 12 \\
OA & 12 & 6 & 6 & $21-55$ & 22 \\
\hline
\end{tabular}

TMD, temporomandibular disorders; TMJ, temporomandibular joints; ADDwR, anterior disc displacement with reduction group; ADDw/oR, anterior disc displacement without reduction group; OA, osteoarthritis.

Western blots were analyzed by densitometry using Image $\mathrm{J}$ software, version $1.47 \mathrm{c}$ (National Institutes of Health, Bethesda, MD, USA).

Immunocytochemistry and confocal microscopy. Immunocytochemistry was performed as previously described (22). Briefly, the synovial fibroblasts $1 \times 10^{6} /$ well were plated in six-well culture plates that contained carry sheet glass. Once the cells on the glass slide had reached $70 \%$ confluence, they were incubated with a mixture of the IL- $1 \beta$ or rhDKK-1 and serum-deprived DMEM. Following $24 \mathrm{~h}$ incubation, the cells were fixed for 10 min with $4 \%$ formaldehyde in phosphate-buffered saline (PBS; $\mathrm{pH}$ 8.0). Following three washes with PBS, the cells were permeabilized for $5 \mathrm{~min}$ with $0.5 \%$ Triton X-100 (Sigma-Aldrich, St. Louis, MO, USA) in PBS, prior to being thoroughly washed again with PBS. PBS supplemented with $10 \%$ FBS was used to block non-specific binding for $60 \mathrm{~min}$. The cells were subsequently incubated with HIF-1 $\alpha$ (1:100) and DKK-1 antibodies (1:50) overnight at $4^{\circ} \mathrm{C}$. The cells were washed thoroughly three times with PBS, and then incubated with $\mathrm{Cy} 3$-conjugated donkey anti-mouse antibody (1:200; Jackson ImmunoResearch Laboratories, Inc., West Grove, PA, USA) at room temperature for $60 \mathrm{~min}$, followed by further washing three times with PBS. The nuclei were then stained with DAPI, and images of the cells were captured and analyzed by CLSM-310 confocal laser scanning microscopy (Carl Zeiss Inc., Thornwood, NY, USA).

Reverse transcription-quantitative polymerase chain reaction (RT-qPCR). TRIzol ${ }^{\circledR}$ reagent (Invitrogen Life Technologies) was used to extract total RNA, according to the manufacturer's instructions. The reaction conditions were as follows: $95^{\circ} \mathrm{C}$ for $5 \mathrm{~min}$, then $95^{\circ} \mathrm{C}$ for $30 \mathrm{sec}, 60^{\circ} \mathrm{C}$ for $30 \mathrm{sec}$ and $72^{\circ} \mathrm{C}$ for $30 \mathrm{sec}$ for 35 cycles, $72^{\circ} \mathrm{C}$ for $10 \mathrm{~min}$ and $15^{\circ} \mathrm{C}$ for $\infty$. The purity and yield of the isolated RNA were determined using an ND 1000 NanoDrop spectrophotometer (Thermo Fisher Scientific, Inc., Wilmington, DE, USA). Aliquots of total RNA were reverse-transcribed using a SYBR PrimeScript ${ }^{\mathrm{TM}}$ RT Reagent kit (Takara Biotechnology Co., Ltd., Dalian, China). RT-qPCR was performed using a SYBR PrimeScript ${ }^{\mathrm{TM}}$ RT-PCR kit (Takara Biotechnology Co., Ltd.) and an ABI PRISM 7500 Real-Time PCR system (Applied Biosystems Life Technologies, Foster City, CA, USA). $\beta$-actin was used as an internal control. The following gene-specific primers were used for the reaction: Human VEGF forward, 5'-CCCACTGAGGAGTCCAACAT-3', and reverse, 5'-TTATACCGGGATTTCTTGCG-3'; human HIF-1 $\alpha$ forward, 5'-CAGGACACAGATTTAGACTGGG-3', and reverse, 5'-TGGTAGTGGTGGCATTAGC-3'; human DKK-1 forward, 5'-GAGTCCTTCTGAGATGATGG-3', and reverse, 5'-TTGATAGCGTTGGAATTGAG-3'; and $\beta$-actin forward, 5'-CCATCGTCCACCGCAAAT-3', and reverse, 5'-CCTGTAACAACGCATCTCATA-3' Primers were purchased from (Sangon Biotech Co. Ltd., Shanghai, China). $\beta$-actin was used as an internal control. The results were obtained as threshold cycle $(\Delta \mathrm{Ct})$ values. No amplification occurred in the non-template controls. The $2^{\Delta \Delta C t}$ method was used to analyze the mRNA expression levels (23).

Wound-healing assay, Boyden chamber migration assay, and tube formation assay. Conditioned medium (CM) from synovial fibroblasts treated with either DKK-1 and HIF-1 $\alpha$ siRNA, or with DKK-1 alone was harvested. HUVECs $\left(2 \times 10^{6} /\right.$ well $)$ were plated in six-well culture plates and grown to $90 \%$ confluence. A scratch was made across the cell layer using a sterile pipette tip to simulate wound healing. The cells then received the indicated culture supernatants. Following $12 \mathrm{~h}$ incubation, the cells were fixed and stained using acridine orange (Sigma-Aldrich), and cells that had migrated into the gap were counted under a microscope (CLSM-310; Carl Zeiss AG, Jena, Germany). Transwell Boyden chambers (Corning Life Sciences, Corning, NY, USA) containing a $6.5 \mathrm{~mm}$ diameter polycarbonate filter ( $8 \mu \mathrm{m}$ pore size) were used to determine the motility of endothelial cells. Serum-starved HUVECs $\left(5 \times 10^{4}\right.$ cells/well) were plated in the upper wells, whereas the indicated culture supernatants, used as a chemo-attractants, were added to the lower wells. The cells were then incubated for $12 \mathrm{~h}$ at $37^{\circ} \mathrm{C}$. Using a cotton swab, the HUVECs on the upper surface were carefully removed, and the cells that had adhered to the underside of the membrane were fixed with $4 \%$ formaldehyde, stained with crystal violet (Beyotime Institute of Biotechnology), and observed under a microscope (BHS-313; Olympus, Tokyo, Japan). For the capillary-like tube formation assay, the HUVECs $\left(1 \times 10^{4} /\right.$ well) were seeded into 96-well plates coated with Matrigel (BD Biosciences, San Jose, CA, USA) in culture supernatants. The cells were then cultured for $6 \mathrm{~h}$ at $37^{\circ} \mathrm{C}$, and the capillary-like structures were counted under a microscope (BHS-313 Olympus).

Statistical analysis. GraphPad Prism 5.00 for Windows (GraphPad Software, Inc., La Jolla, CA, USA) was used for statistical analysis. A Pearson correlation analysis was used to 
determine the correlation between DKK-1 and VEGF expression. Student's t-tests were performed to analyze the results of HIF-1 $\alpha$ nuclear translocation. A one-way analysis of variance with post-Tukey analysis was used to analyze the results of the wound-healing assay, transwell assay, tube formation, and RT-qPCR. All data were presented as the mean \pm standard error of the mean. $\mathrm{P}<0.05$ was considered to indicate a statistically significant difference.

\section{Results}

Elevated expression levels of DKK-1 are associated with increased expression levels of VEGF in the synovial fluid of patients with TMD. To determine the expression levels of DKK-1 in the synovial fluid of patients with TMD, the synovial fluid from 50 patients and seven healthy controls were collected for analysis. The expression levels of DKK-1 in the patients with TMD were significantly higher, as compared with the control subjects $(\mathrm{P}<0.01$ Fig. $1 \mathrm{~A})$. The expression levels of DKK-1 in the TMD-OA group were significantly higher, as compared with those of the TMD-ADDwR and TMD-ADDw/oR groups $(\mathrm{P}<0.05)$, and with those of the control group $(\mathrm{P}<0.001$, Fig. 1B). The expression levels of DKK-1 in the synovial fluid of the TMD-ADDw/oR group was significantly increased, as compared with those of the control group $(\mathrm{P}<0.05$, Fig. 1B). However, the difference between the mean expression levels of DKK-1 in patients with TMD-ADDwR and the control subjects was not significant (Fig. 1B). As shown in Fig. 1C, the expression levels of VEGF in the synovial fluid of patients with TMD was significantly higher, as compared with the control group $(\mathrm{P}<0.001)$. The expression levels of VEGF in the OA group were significantly higher, as compared with the ADD groups $(\mathrm{P}<0.05)$ and control group $(\mathrm{P}<0.001$, Fig. 1D). The expression levels of VEGF in the TMD-ADDwR group and TMD-ADDw/oR group were not significantly different, as compared with the control group (data not shown). Furthermore, the expression levels of DKK-1 were positively associated with higher expression levels of VEGF $(\mathrm{P}<0.05, \mathrm{r}=0.2947$, $\mathrm{n}=63$, Fig. 1E). These results suggest that the expression levels of DKK-1 are significantly increased in the synovial fluid of patients with TMD, and that DKK-1 expression may have an important role in TMD angiogenesis.

DKK-1 increases the expression levels of inflammation-induced VEGF in synovial fibroblasts. To assess the expression levels of DKK-1 in inflammatory primary synovial fibroblasts, the fibroblasts were initially stimulated with inflammatory IL-1 $\beta$. Previous studies have suggested that the expression levels of IL-1 $\beta$ are increased in the synovial fluid of TMD patients, and that IL- $1 \beta$ is regarded as a key inflammatory response regulator $(3,19,24)$. Following treatment with IL-1 $\beta$, both the mRNA and protein expression levels of DKK-1 increased, as compared with the control (Fig. 2A-C). The fluorescence intensity of DKK-1 in the synovial fibroblasts following treatment with IL-1 $\beta$ are shown in Fig. 2B. Images of each group (0. 5, and $10 \mu \mathrm{g} / \mathrm{ml} \mathrm{IL-1 \beta )}$ were captured using the same parameters. In the untreated group, fluorescence intensity was lower, as compared with that of the IL-1 $\beta$-treated group. The expression levels of DKK-1 were dose-dependently elevated in both the cytoplasm and nucleus of the synovial fibroblasts following treatment with IL-1 $\beta$. These results were concordant with the results obtained from the ELISA. The present study also detected the expression levels of VEGF following treatment with IL-1 $\beta$. IL-1 $\beta$ dose-dependently increased both the protein and mRNA expression levels of VEGF (Fig. 2A and C). To determine whether DKK-1 increased the expression levels of VEGF, the synovial fibroblasts were co-treated with IL-1 $\beta$ and anti-DKK-1 antibody. Treatment with anti-DKK-1 antibody inhibited the stimulatory effects of IL-1 $\beta$ on VEGF protein expression (Fig. 2D). Furthermore, the expression levels of VEGF were induced by rhDKK-1. These results suggest that DKK-1 is upregulated during inflammation, and that DKK-1 induces VEGF expression in synovial fibroblast cells.

HIF-1 $\alpha$ mediates DKK-1 regulation of VEGF expression. To investigate the mechanisms underlying DKK-1 regulation of VEGF expression, the expression levels of HIF-1 $\alpha$, an upstream inducer of VEGF, were analyzed. HIF- $1 \alpha$ siRNA 2 markedly inhibited the expression of HIF-1 $\alpha$ (Fig. 3A). HIF-1 $\alpha$ siRNA 2 was therefore chosen as the target sequence. As shown in Fig. 3B, the protein expression levels of HIF-1 $\alpha$ were increased in synovial fibroblasts treated with rhDKK-1, as compared with the negative control. The protein expression levels of VEGF exhibited similar results. However, the upregulatory effects of rhDKK-1 on VEGF and HIF-1 $\alpha$ protein expression were inhibited by HIF-1 $\alpha$ siRNA (Fig. 3B). The mRNA expression levels of HIF-1 $\alpha$ and VEGF were also analyzed (Fig. 3C and D). The mRNA expression levels of VEGF were concordant with its protein expression levels (Fig. 3C). Notably, no significant differences in the mRNA expression levels of HIF-1 $\alpha$ were observed between the negative control group and the rhDKK-1 treatment group (Fig. 3D). These results suggest that DKK-1 may increase HIF-1 $\alpha$ protein translation or decrease HIF-1 $\alpha$ degradation, rather than upregulate the transcription levels of HIF-1 $\alpha$. The nuclear localization of HIF-1 $\alpha$ was also investigated. As shown in Fig. 3E and F, rhDKK-1 significantly increased HIF-1 $\alpha$ nuclear translocation, as compared with the untreated group. These results indicate that HIF-1 $\alpha$ translocates to the nucleus following DKK-1 exposure, and mediates DKK-1-induced VEGF expression.

HIF-1 $\alpha$ siRNA inhibits DKK-1-induced elevation of HUVEC migration. The synovial fibroblasts were treated with rhDKK-1 and/or HIF-1 $\alpha$ siRNA, following which the CM was collected and, the cells were cultured in fresh serum-deprived medium for $24 \mathrm{~h}$. To further assess the biological role of DKK-1 in angiogenesis, the effects of DKK-1 on HUVECs were investigated using a wound healing assay. As shown in Fig. 4A, treatment of the HUVECs with CM from synovial fibroblasts pretreated with rhDKK-1 increased migration 6 -fold, as compared with the control. Furthermore, similar results were observed in the transwell and tube formation assays (Fig. 4A-D). In order to reveal the precise molecular mechanism underlying DKK-1-induced angiogenesis induction, the present study investigated whether HIF-1 $\alpha$, a key regulator of angiogenesis, mediated DKK-1-induced endothelial cell function. As expected, the CM from the synovial 
A

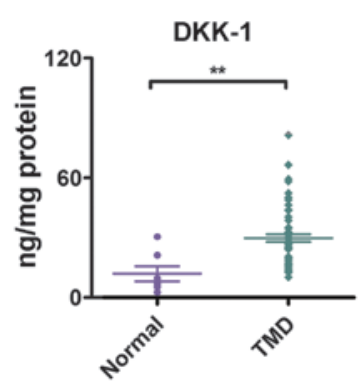

D

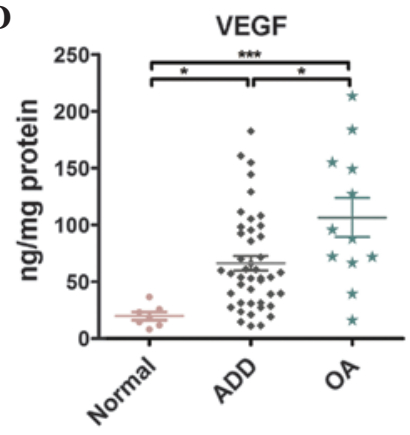

$\mathbf{B}$

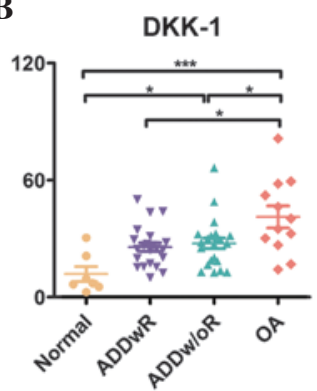

$\mathbf{E}$

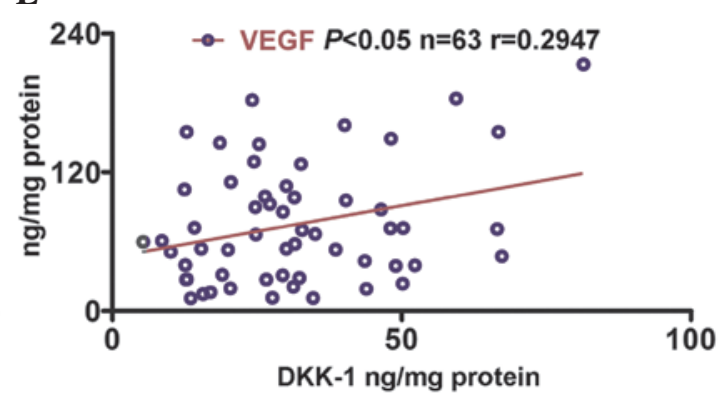

C

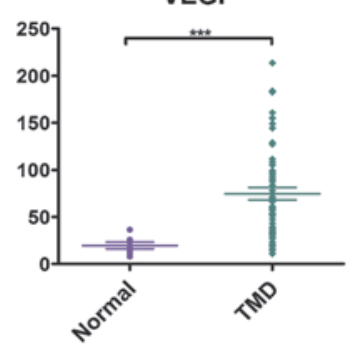

100

Figure 1. Elevated expression levels of dickkopf-related protein 1 (DKK-1) are associated with increased expression levels of vascular endothelial growth factor (VEGF) in the synovial fluid of patients with temporomandibular joint disorder (TMD). (A) The expression levels of DKK-1 in both the control group $(\mathrm{n}=7)$ and TMD group $(\mathrm{n}=56) ;{ }^{* *} \mathrm{P}<0.01$; Student's t-test. (B) The expression levels of DKK-1 in the control group $(\mathrm{n}=7)$ and the following patient groups: The anterior disc displacement with reduction (ADDwR, $\mathrm{n}=22$ ), the anterior disc displacement without reduction (ADDw/oR, $\mathrm{n}=22$ ), and the osteoarthritis $(\mathrm{OA}$, $\mathrm{n}=12$ ) group [ $\mathrm{P}<0.05$, and ${ }^{* * *} \mathrm{P}<0.001$; two-way analysis of variance (ANOVA)]. (C) The expression levels of VEGF in both the control group ( $\mathrm{n}=7$ ) and TMD group ( $\mathrm{n}=56$ samples); ${ }^{* * *} \mathrm{P}<0.001$; Student's t-test. (D) The expression levels of VEGF in the control group $(\mathrm{n}=7)$ and the following patient groups: The anterior disc displacement (ADD, $\mathrm{n}=44)$ and the OA group $(\mathrm{n}=12)\left({ }^{*} \mathrm{P}<0.05\right.$, and ${ }^{* * * *} \mathrm{P}<0.001$; two-way ANOVA). (E) Association between the expression levels of DKK-1 and VEGF, as determined by a Pearson correlation test.

A

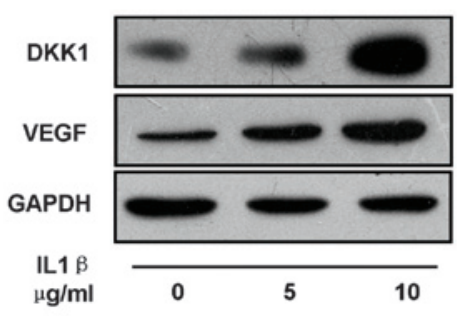

C

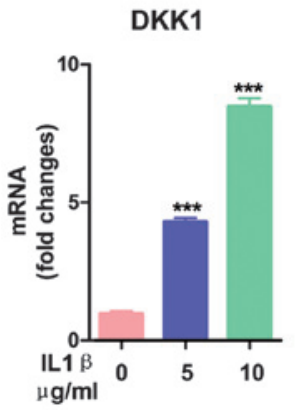

B

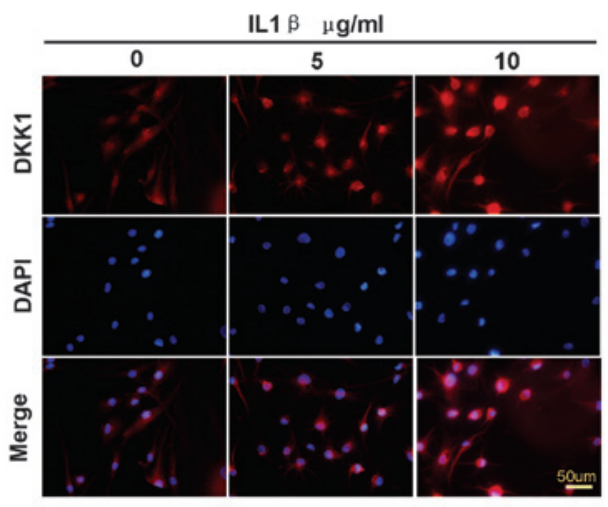


A

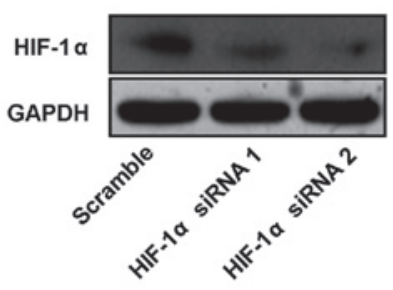

B

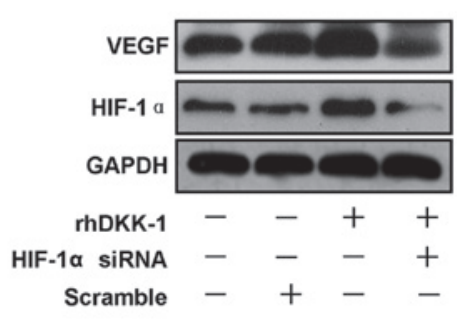

$\mathbf{C}$

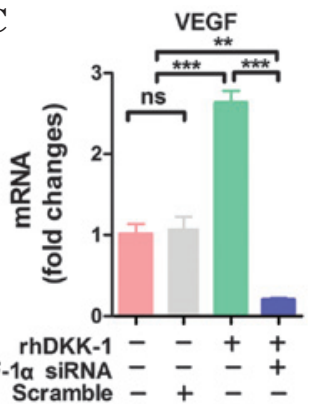

D

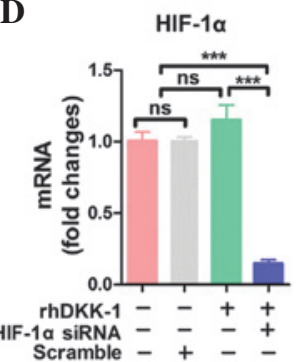

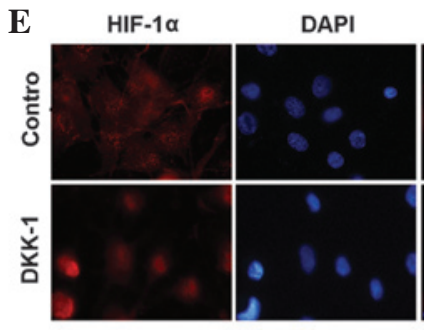

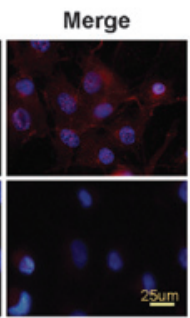

$\mathbf{F}$

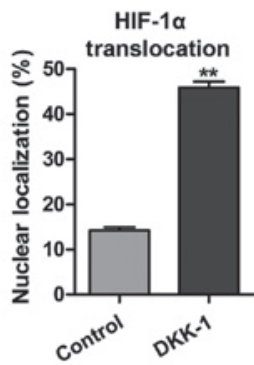

Figure 3. Hypoxia inducible factor-1 $\alpha$ (HIF-1 $\alpha$ ) mediates dickkopf-related protein 1 (DKK-1) regulation of vascular endothelial growth factor (VEGF) expres-

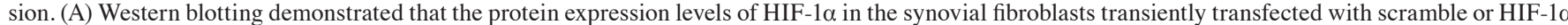
small interfering (si)RNA 1 and 2. The proteins were extracted $72 \mathrm{~h}$ post-transfection. (B) HIF-1 $\alpha$ siRNA (HIF-1 $\alpha$ siRNA 2 as a target sequence) significantly inhibited the upregulatory effects of recombinant human (rh)DKK-1 on VEGF protein expression in synovial fibroblasts, as demonstrated by western blot analysis. (C and D) HIF-1 $\alpha$ siRNA also inhibited the upregulatory effects of rhDKK-1 on the mRNA expression levels of VEGF in synovial fibroblasts. The mRNA expression levels of HIF-1 $\alpha$ exhbited no significant changes between the control and rhDKK-1 treatment group. The results are presented as the mean \pm standard error of the mean. ${ }^{* *} \mathrm{P}<0.01$, and ${ }^{* * *} \mathrm{P}<0.001$; one-way analysis of variance. RNA was extracted $48 \mathrm{~h}$ post-transfection. (E) Synovial fibroblasts treated with $10 \mathrm{ng} / \mathrm{ml}$ rhDKK-1 for $24 \mathrm{~h}$ exhibited HIF-1 $\alpha$ nuclear translocation, as determined by immunofluorescence. Scale bars, $25 \mu \mathrm{m}$. (F) Quantification of nuclear translocation of HIF-1 $\alpha$ in the vehicle group and rhDKK-1-treatment group. The results are presented as the mean \pm standar error of the mean, ${ }^{* *} \mathrm{P}<0.01 ;$ Student's t-test.
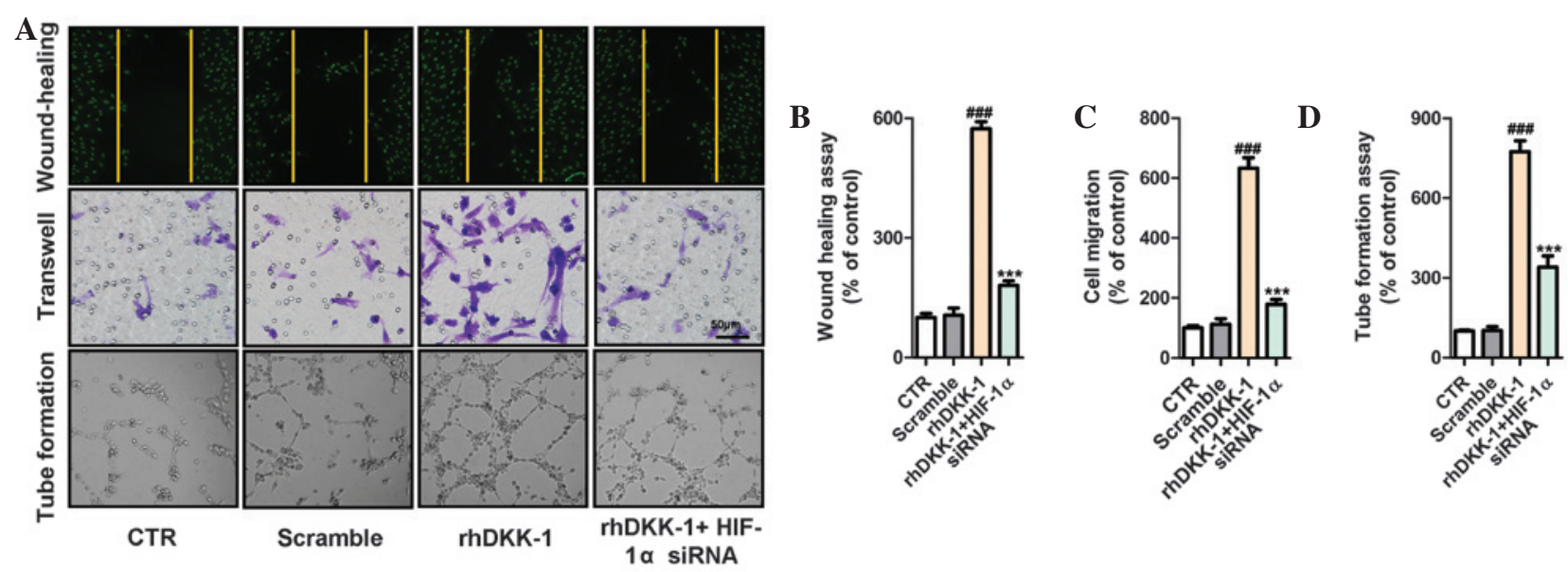

Figure 4. Hypoxia inducible factor-1 $\alpha$ (HIF-1 $\alpha$ ) small interfering (si)RNA inhibits dickkopf-related protein 1 (DKK-1)-induced increase of human umbilical vein endothelial cells (HUVEC) migration. To collect the conditioned medium (CM), the synovial fibroblasts were treated with the indicated agents, prior to being cultured in fresh serum-deprived medium for $24 \mathrm{~h}$, followed by collection of the CM. (A) Recombinant human (rh)DKK-1 accelerated the migration of endothelial cells as visualized by the wound-healing, transwell ( $8 \mu \mathrm{m}$ pore size; scale bars, $50 \mu \mathrm{m})$, and tube formation assays. Pre-treatment with HIF- $1 \alpha$ siRNA significantly inhibited the upregulatory effects of rhDKK-1. Quantification of HUVEC migration and tube formation in each group. The data are presented as the mean \pm standard error of the mean. ${ }^{\# \# "} \mathrm{P}<0.001$, vs. the control group (CTR) or scramble group, and ${ }^{* * *} \mathrm{P}<0.001$, vs. the rhDKK-1 treatment group; one-way analysis of variance. (B) Wound-healing assay, (C) transwell assay, and (D) tube formation assay.

fibroblasts pretreated with HIF-1 $\alpha$ siRNA significantly suppressed HUVEC migration, as compared with rhDKK-1 treatment alone, or scramble alone. These results were also demonstrated by the wound-healing assay, transwell assay, and tube formation (Fig. 4A-D).

\section{Discussion}

DKK-1 is a potent secreted Wnt antagonist, which is a member of the DKK family that includes DKK-1, 2, 3, 4 and the DKK3-related protein, Soggy $(25,26)$. DKK family members 
are implicated in various developmental, pathological, and physiological processes $(16,27)$. In prostate cancer, DKK-1 acts as a marker of carcinogenesis, and may provide a novel method of prostate cancer prediction for patients with negative primary biopsy results, who may otherwise progress to bone metastasis (28). Recent studies have demonstrated that overexpression of DKK-1 may be associated with increased angiogenesis $(14,15)$. However, the mechanism underlying the role of DKK-1 in the process of angiogenesis remains to be elucidated. The results of the present study demonstrated that DKK-1 promotes angiogenic factor secretion in synovial fibroblasts from patients with TMD, and promotes migration of endothelial cells. The present study provided, to the best of our knowledge, the first evidence that DKK-1 is highly expressed in the synovial fluid of patients with TMD, and is positively correlated with VEGF expression. Treatment of the synovial fibroblasts with rhDKK-1 and anti-DKK-1 demonstrated that VEGF expression was regulated by DKK-1. In addition HIF-1 $\alpha$ nuclear localization was implicated in the DKK-1/VEGF axis.

A previous study demonstrated that VEGF is involved in TMD (9). VEGF is a selective endothelial mitogen and vascular permeability factor, which is upregulated in patients with chronic closed lock (CCL) of the TMJ, and the expression levels of VEGF in the synovial fluid of patients with TMD reflects the clinical status of patients with CCL. Furthermore, VEGF may be an important molecular target for future chemotherapy treatment of TMD and CCL (29). Our previous study demonstrated that expression of VEGF121 and VEGF165 in synovial fibroblasts promoted inflammatory angiogenesis in TMD (9). These results suggest that VEGF has an important role in TMD initiation and progression, and further elucidates the molecular mechanism underlying VEGF upregulation. The results of the present study indicated that VEGF expression was induced by $\mathrm{rhDKK}-1$, and downregulated by anti-DKK-1. In numerous cell types, HIF-1 $\alpha$ regulates VEGF expression (30-32). Notably, the results of our studies have also suggested that VEGF is associated with the activation of HIF-1 $\alpha$. DKK-1, in the present study, caused a rapid increase in the protein expression levels of HIF-1 $\alpha$. The results of the present study also demonstrated that there was no significant difference in the mRNA expression levels of HIF-1 $\alpha$ between the negative control group and the rhDKK-1 treatment group; these effects are likely due to DKK-1-induced protection of HIF- $1 \alpha$ stability, and promotion of HIF- $1 \alpha$ nuclear translocation in the synovial fibroblasts, rather than an increase in HIF-1 $\alpha$ transcription. Therefore, DKK-1-induced HIF-1 $\alpha$ upregulation in the synovial fibroblasts of TMJ, may be associated with HIF-1 $\alpha$ protein stability.

In the present study, rhDKK-1 was able to induce endothelial cell migration and chemotaxis, which are important processes in angiogenesis. Furthermore, pretreatment with HIF-1 $\alpha$ siRNA significantly inhibited rhDKK-1-induced HUVEC migration and chemotaxis. A previous study reported that DKK-1 was able to promote angiogenesis of endothelial progenitor cells through $\mathrm{C}-\mathrm{X}-\mathrm{C}$ chemokine receptor type 4 and VEGF receptor 2 signaling (17). DKK-1 is a regulator of vasculogenic progenitors that enhance neovascularization in Matrigel plugs (33). The present study demonstrated that knockdown of HIF-1 $\alpha$ was able to decrease DKK-1-induced enhancement of angiogenic factor expression. These results were concordant with the hypothesis that DKK-1 promotes angiogenesis in TMD. However, the present study carried out these investigations in synovial fibroblasts alone, and DKK-1 also promotes the upregulation of VEGF expression via HIF-1 $\alpha$ in a classic angiogenesis pathway. Achieving further understanding of the molecular mechanism underlying DKK-1 promotion of angiogenesis in TMD may provide the basis of a treatment strategy that may delay or halt TMD progression. This mechanism requires further investigation.

In conclusion, the present study demonstrated that DKK-1 is highly expressed in the synovial fluid of patients with TMD, and DKK-1 expression is positively correlated with VEGF expression. The results of the present study also demonstrated that upregulation of DKK-1 expression is associated with angiogenic activities in the synovial fluid of TMD. Furthermore, HIF-1 $\alpha$ was associated with DKK-1-induced HUVEC functional activation. The present study provides further understanding regarding the mechanism underlying TMD development.

\section{Acknowledgements}

The present study was supported by grants from the National Natural Science Foundations of China to Professor Xing Long (grant no. 81271171), and Professor Wei Fang (grant no. 81200804). The authors of the present study are grateful to EssayStar for its linguistic assistance during the preparation of the manuscript.

\section{References}

1. Aliko A, Ciancaglini R, Alushi A, Tafaj A and Ruci D: Temporomandibular joint involvement in rheumatoid arthritis, systemic lupus erythematosus and systemic sclerosis. Int J Oral Maxillofac Surg 40: 704-709, 2011.

2. de Boer EW, Dijkstra PU, Stegenga B, de Bont LG and Spijkervet FK: Value of cone-beam computed tomography in the process of diagnosis and management of disorders of the temporomandibular joint. Br J Oral Maxillofac Surg 52: 241-246, 2014.

3. Satoh K, Ogura N, Akutsu M, Kuboyama N, Kuyama K, Yamamoto $\mathrm{H}$ and Kondoh T: Expression of cyclooxygenase-1 and -2 in IL-1beta-induced synovitis of the temporomandibular joint. J Oral Pathol Med 38: 584-590, 2009.

4. Varol A, Basa S, Topsakal A and Akpinar I: Assessment of synovial vascularization by power Doppler ultrasonography in TMJ internal derangements treated arthroscopically. Br J Oral Maxillofac Surg 46: 625-630, 2008.

5. Camejo Fde A, Almeida LE, Doetzer AD, Caporal KS, Ambros V, Azevedo M, Alanis LR, Olandoski M, Noronha L and Trevilatto PC: FasL expression in articular discs of human temporomandibular joint and association with osteoarthrosis. J Oral Pathol Med 43: 69-75, 2014.

6. Sato J, Segami N, Nishimura M, Yoshimura H, Demura N, Yoshitake Y and Nishikawa K: Correlation between the arthroscopic diagnosis of synovitis and microvessel density in synovial tissues in patients with internal derangement of the temporomandibular joint. J Craniomaxillofac Surg 31: 101-106, 2003.

7. Carmeliet P and Jain RK: Molecular mechanisms and clinical applications of angiogenesis. Nature 473: 298-307, 2011.

8. Dejana E and Lampugnani MG: Differential adhesion drives angiogenesis. Nat Cell Biol 16: 305-306, 2014.

9. Ke J, Liu Y, Long X, Li J, Fang W, Meng Q and Zhang Y: Up-regulation of vascular endothelial growth factor in synovial fibroblasts from human temporomandibular joint by hypoxia. J Oral Pathol Med 36: 290-296, 2007.

10. Song J, Cao L and Li Y: RNA interference-mediated inhibition of survivin and VEGF in pancreatic cancer cells in vitro. Mol Med Rep 7: 1651-1655, 2013. 
11. Gao W, Sweeney C, Connolly M, Kennedy A, Ng CT, McCormick J, Veale DJ and Fearon U: Notch-1 mediates hypoxia-induced angiogenesis in rheumatoid arthritis. Arthritis Rheum 64: 2104-2113, 2012

12. Liao HY, Wang GP, Huang SH, Li Y, Cai SW, Zhang J, Chen HG and $\mathrm{Wu}$ WB: HIF-1 $\alpha$ silencing suppresses growth of lung adenocarcinoma A549 cells through induction of apoptosis. Mol Med Rep 9: 911-915, 2014.

13. She Q, Xia S, Deng SB, Du JL, Li YQ, He L, Xiao J and Xiang YL: Angiogenesis in a rat model following myocardial infarction induced by hypoxic regulation of $\mathrm{VEGF}_{165}$ gene-transfected EPCs. Mol Med Rep 6: 1281-1287, 2012.

14. Park H, Jung HY, Choi HJ, Kim DY, Yoo JY, Yun CO, Min JK, Kim YM and Kwon YG: Distinct roles of DKK1 and DKK2 in tumor angiogenesis. Angiogenesis 17: 221-234, 2014.

15. Weng LH, Ko JY, Wang CJ, Sun YC and Wang FS: Dkk-1 promotes angiogenic responses and cartilage matrix proteinase secretion in synovial fibroblasts from osteoarthritic joints. Arthritis Rheum 64: 3267-3277, 2012.

16. Zhang H, Yu C, Dai J, Keller JM, Hua A, Sottnik JL, Shelley G, Hall CL, Park SI, Yao Z, et al: Parathyroid hormone-related protein inhibits DKK-1 expression through c-Jun-mediated inhibition of $\beta$-catenin activation of the DKK-1 promoter in prostate cancer. Oncogene 33: 2464-2477, 2014

17. Smadja DM, d'Audigier C, Weiswald LB, Badoual C, Dangles-Marie V, Mauge L, Evrard S, Laurendeau I, Lallemand F, Germain S, et al: The Wnt antagonist Dickkopf-1 increases endothelial progenitor cell angiogenic potential. Arterioscler Thromb Vasc Biol 30: 2544-2552, 2010.

18. Dworkin SF and LeResche L: Research diagnostic criteria for temporomandibular disorders: Review, criteria, examinations and specifications, critique. J Craniomandib Disord 6: 301-355, 1992.

19. Kostrzewa-Janicka J, Jurkowski P, Nedzi-Gora M and Mierzwinska-Nastalska E: Inflammatory markers in temporomandibular joint disorders. Cent Eur J Immunol 37: 290-293, 2012

20. Cai HX, Luo JM, Long X, Li XD and Cheng Y: Free-radical oxidation and superoxide dismutase activity in synovial fluid of patients with temporomandibular disorders. J Orofac Pain 20: 53-58, 2006.

21. Li J, Long X, Ke J, Meng QG, Lee WC, Doocey JM and Zhu F: Regulation of HAS expression in human synovial lining cells of TMJ by IL-1beta. Arch Oral Biol 53: 60-65, 2008.

22. Zhang L, Sun ZJ, Bian Y and Kulkarni AB: MicroRNA-135b acts as a tumor promoter by targeting the hypoxia-inducible factor pathway in genetically defined mouse model of head and neck squamous cell carcinoma. Cancer Lett 331: 230-238, 2013.
23. Wu Y, Zhu L, Liu L, Zhang J and Peng B: Interleukin-17A stimulates migration of periodontal ligament fibroblasts via p38 MAPK/NF- $\kappa$ B-dependent MMP-1 expression. J Cell Physiol 229: 292-299, 2014

24. Akutsu M, Ogura N, Ito K, Kawashima M, Kishida T, and Kondoh T: Effects of interleukin- $1 \beta$ and tumor necrosis factor- $\alpha$ on macrophage inflammatory protein-3 $\alpha$ production in synovial fibroblast-like cells from human temporomandibular joints. J Oral Pathol Med 42: 491-498, 2013.

25. Choi HJ, Park H, Lee HW and Kwon YG: The Wnt pathway and the roles for its antagonists, DKKS, in angiogenesis. IUBMB Life 64: 724-731, 2012.

26. Min JK, Park H, Choi HJ, Kim Y, Pyun BJ, Agrawal V, Song BW, Jeon J, Maeng YS, Rho SS, et al: The WNT antagonist Dickkopf2 promotes angiogenesis in rodent and human endothelial cells. J Clin Invest 121: 1882-1893, 2011

27. Jung IL, Kang HJ, Kim KC and Kim IG: Knockdown of the Dickkopf 3 gene induces apoptosis in a lung adenocarcinoma. Int J Mol Med 26: 33-38, 2010.

28. D'Amelio P, Roato I, Oderda M, Soria F, Zitella A, Ferracini R, Mengozzi G, Gontero P and Isaia GC: DKK-1 in prostate cancer diagnosis and follow up. BMC Clin Pathol 14: 11, 2014.

29. Kumagai K, Hamada Y, Holmlund AB, Gotoh A, Nakaoka K, Arai G, Yamane S and Suzuki R: The levels of vascular endothelial growth factor in the synovial fluid correlated with the severity of arthroscopically observed synovitis and clinical outcome after temporomandibular joint irrigation in patients with chronic closed lock. Oral Surg Oral Med Oral Pathol Oral Radiol Endod 109: 185-190, 2010.

30. Trisciuoglio D, Gabellini C, Desideri M, Ragazzoni Y, De Luca T, Ziparo E and Del Bufalo D: Involvement of BH4 domain of bcl-2 in the regulation of HIF-1-mediated VEGF expression in hypoxic tumor cells. Cell Death Differ 18: 1024-1035, 2011.

31. Vadlapatla RK, Vadlapudi AD, Pal D, Mukherji M and Mitra AK: Ritonavir inhibits HIF-1 $\alpha$-mediated VEGF expression in retinal pigment epithelial cells in vitro. Eye (Lond) 28: 93-101, 2014.

32. Huang L, Zhang Z, Zhang S, Ren J, Zhang R, Zeng H, Li Q and $\mathrm{Wu}$ G: Inhibitory action of Celastrol on hypoxia-mediated angiogenesis and metastasis via the HIF-1 $\alpha$ pathway. Int J Mol Med 27: 407-415, 2011.

33. Aicher A, Kollet O, Heeschen C, Liebner S, Urbich C, Ihling C, Orlandi A, Lapidot T, Zeiher AM and Dimmeler S: The Wnt antagonist Dickkopf-1 mobilizes vasculogenic progenitor cells via activation of the bone marrow endosteal stem cell niche. Circ Res 103: 796-803, 2008. 